\title{
Prueba de micronúcleo en mucosa para la evaluación de toxicidad en perros por exposición al tabaco
}

\author{
Mucosal micronucleus test for the evaluation of toxicity in dogs by exposure to tobacco
}

\author{
Alex Alves Rodrigues ${ }^{1}$, Jéssica Cardoso Faria ${ }^{2}$, Andrea Barbosa Pimenta ${ }^{3}$, Jorge Alberto Prieto Perez ${ }^{4}$, \\ Silvio de Almeida Junior ${ }^{5^{*}}$
}

\author{
${ }^{1}$ Faculdade Metropolitana Unidas, graduação em Medicina Veterinária, São Paulo, São Paulo, Brasil. ${ }^{2}$ Euro Anglo Cursos \\ Profissionalizantes, Franca, São Paulo. ${ }^{3}$ Faculdade Sudoeste Paulista, graduação em Medicina Veterinária, Avaré, São Paulo, Brasil. \\ ${ }^{4}$ Universidad Pontificia Bolivariana, Bucaramanga, Santander, Colombia. ${ }^{5}$ Programa de Pós-graduação em Ciência Animal - \\ Universidade de Franca, Franca, São Paulo Brasil. *Autor para correspondência: silvioalmeidajr@yahoo.com.br
}

\begin{abstract}
Resumen: Es necesario estudiar el efecto mutagénico del tabaquismo pasivo en los animales, para que las personas puedan conocer y evitar fumar cerca de sus mascotas. Según la Organización Mundial de la Salud, el humo del cigarrillo es el principal agente de contaminación doméstica y ambiental, teniendo en cuenta que la población general pasa el $80 \%$ de su tiempo en interiores, lo que hace que el estudio sea relevante. Una forma de detección es la rotura cromosómica identificada por el método de análisis de micronúcleos en células epiteliales orales exfoliadas para observar el efecto pro-mutagénico del humo del cigarrillo en la mucosa oral de los perros expuestos al tabaco. Muchos estudios han examinado los efectos del tabaquismo pasivo en humanos, pero se ha estudiado muy poco en relación a los animales domésticos, aunque existe evidencia de un mayor riesgo de cáncer de pulmón en perros con humo pasivo. A partir de esta revisión de la literatura, fue posible observar un efecto pro-mutagénico del humo del cigarrillo en la mucosa oral de los perros expuestos al tabaquismo pasivo.
\end{abstract}

Palabras claves: tabaquismo, células, mutación, cigarrillo, perro, pro-mutágeno.

\begin{abstract}
It is necessary to study the mutagenic effect of passive smoking on animals, so that people can become aware of and avoid smoking near their pets. According to the World Health Organization, cigarette smoke is the largest domestic and environmental pollution agent, considering that the general population spends $80 \%$ of their time indoors, making the study relevant. One form of screening is chromosomal breakage identified by the micronucleus method assay on exfoliated oral epithelial cells to observe the pro-mutagenic effect of cigarette smoke on the oral mucosa of dogs exposed to smoking. Many studies have examined the effects of passive smoking on humans, but little has been studied in relation to domestic animals, although there is evidence of increased risk for lung cancer in passive smoke dogs. From this literature review, it was possible to observe a pro-mutagenic effect of cigarette smoke on the oral mucosa of dogs exposed to passive smoking.
\end{abstract}

Keywords: smoking, cells, mutation, cigarette and dog.

\section{Introducción}

El tabaquismo pasivo se considera actualmente el principal contaminante presente en ambientes interiores, residenciales o comerciales. Del mismo modo, se cita como una de las causas evitables de muertes primarias en los países desarrollados, junto con el tabaquismo activo y el alcoholismo (WHO, 2011). En los Estados Unidos, se estima que hay 3.000 muertes por cáncer de pulmón en "fumadores pa sivos" y 62.000 muertes por enfermedad cardiovascular anualmente, lo que puede correlacionarse con el mismo. En contraste, otros contaminantes ambientales justifican menos de 100 muertes por cáncer de pulmón por año (Santos, 2018). La relación entre el tabaco y el cáncer de pulmón puede estar relacionada con un mayor riesgo de cáncer en otros lugares del cuerpo, como la cabeza y el cuello (esófago, laringe, lengua, glándulas salivales, labios, boca y faringe), vejiga, cuello uterino, mama, páncreas e intestino (Gomes, 2003).

Según la literatura científica, el humo de segunda mano aumenta el riesgo de mutagénesis en humanos, especialmente en niños y ancianos. Por lo tanto, esta revisión se realizó con el objetivo de evaluar el efecto mutagénico en animales domésticos, en los que viven directamente con el grupo de riesgo (tutores). Este es un estudio de literatura retrospectivo para evaluar el uso de la prueba de micronúcleos para medir el potencial mutagénico y la sensibilidad de los perros expuestos pasivamente (Wünsch Filho et al., 2010). 


\section{Revisión}

El cáncer de cavidad oral es parte del conjunto de cánceres de cabeza y cuello, observado en aproximadamente 300,000 casos nuevos en todo el mundo desde 2012, de los cuales aproximadamente dos tercios son hombres. El tipo más común de cáncer de la cavidad oral es el carcinoma de células escamosas, que en general se desarrolla desde la progresión de una hiperplasia epitelial a un carcinoma in situ y luego a la forma invasiva. Sin embargo, no todos los carcinomas pasan por estas etapas (INCA, 2015).

Vinculada a este tipo de tumor, la exposición al humo del cigarrillo perjudica el transporte mucociliar en humanos, tanto en exposiciones agudas (Bascom, 1995) como en exposiciones crónicas (Stanley, 1986; Karaman, 2009), con el humo del cigarrillo asociado a cambios profundos en los mecanismos de producción de moco. Del mismo modo, el humo del cigarrillo puede ocasionar alteraciones funcionales e importantes cambios estructurales en el epitelio respiratorio (Wünsch Filho et al., 2010).

Con respecto a los animales, existe una creciente incidencia de trastornos neoplásicos en perros, principalmente debido a la mayor longevidad observada y otros factores como la nutrición ejemplificada por las dietas equilibradas, las vacunas que actúan en la prevención temprana de enfermedades infecciosas, los métodos precisos del diagnóstico y también los protocolos terapéuticos cada vez más específicos y efectivos que contribuyen a la longevidad de los perros (Withrow \& Macewen, 1996; Maria et al., 1998; Morrison, 1998). Withrow y Macewen (1996) describen al cáncer como una enfermedad relacionada con la senescencia, sin embargo, Sturion et al. (1997) en sus informes diagnosticaron osteocondroma a un perro brasileño de 2 meses.

Con respecto a la predisposición como resultado de la raza, Gilson y Page (1998) encontraron que los mastocitomas afectan principalmente a los perros braquicéfalos y los tumores óseos son más frecuentes en las razas grandes y gigantes. En cuanto al género, Maria et al. (1998) observaron una mayor prevalencia de neoplasias en las mujeres, aproximadamente el $71 \%$ y el $75 \%$, respectivamente, en comparación con los hombres.

Con respecto a la incidencia, Gorman y Dobson (1995) encontraron que las neoplasias cutáneas se diagnosticaban con mayor frecuencia, en comparación con los tumores de otros órganos, que representan aproximadamente el 30\% de las neoplasias en perros. De manera similar, Withrow (1996) afirmó que los tumores de piel y tejido subcutáneo son los más comunes en perros, representando aproximadamente $1 / 3$ de todas las neoplasias diagnosticadas en esta especie (mastocitos, lipoma, adenoma de glándulas sebáceas, melanoma, hemangiopericitoma). Otra neoplasia en crecimiento es el carcinoma de células escamosas, un tumor maligno relacionado con su patogénesis con la exposición a los rayos UV (Rodrigues \& Junior, 2019).

Sin embargo, las neoplasias pulmonares se diagnostican constantemente en la rutina clínica y, debido a su sintomatología inespecífica, su diagnóstico puede no realizarse inadvertidamente, o retrasarse perjudicialmente, lo que implica numerosas hipótesis de diagnóstico diferencial (Armor et al., 1998).

\section{Discusión}

La presencia de micronúcleos en las células exfoliativas de la mucosa yugal refleja el daño genotóxico de la capa basal epitelial (Carrard et al., 2007) y puede o no estar relacionado con la formación de tumores, más indicativo de cambios (Carvalho et al., 2002). Por lo tanto, a partir de la información encontrada en la literatura y los resultados obtenidos por Moralles (2014) es posible afirmar y observar que el efecto del tabaquismo afecta no solo al fumador activo sino a todos los individuos en su vida, incluidos los animales.

Meireles et. al. (2006), mostraron en su estudio la alteración de las células exfoliadas de la mucosa oral en pacientes directamente expuestos, mientras que Wunsch Filho et al. (2010) demuestran a través de un estudio epidemiológico el índice de cambios en humanos (ancianos y niños principalmente) afectad os por tumores en la laringe, faringe y cavidad oral, que fueron expuestos pasivamente al tabaquismo. Según la WHO, el tabaquismo pasivo se define como la inhalación de humo y productos de tabaco por parte de no fumadores pero que viven con fumadores en el interior (INCA, 2015).

Moralles (2014) evaluó el efecto mutagénico inducido por el tabaquismo pasivo mediante el uso de grupos de perros mediante análisis de micronúcleos en células de la mucosa yugal. El uso de pruebas de micronúcleos para detectar y cuantificar cambios genotóxicos y mutagénicos por numerosos agentes cancerígenos se ha establecido en estudios in vivo e in vitro en animales y plantas (Sisenando, 2011). La sensibilidad de la prueba de micronúcleos es comparable a los estudios genéticos de roturas y translocaciones cromosómicas (Schmid, 1975), y esta prueba se desarrolló como un método citogenético cuantitativo para evaluar la frecuencia de daño cromosómico in vivo en células nucleadas (Silva, 2012). 
La metodología de investigación de micronúcleos en células exfoliativas / epiteliales se describió previamente como efectiva para la detección de mutaciones adquiridas o inducidas debido a la alteración del material genético con roturas cromosómicas (Dietz et al., 2000). Para ser clasificado como un micronúcleo, se debe identificar una estructura de cromatina similar y una intensidad de color similar o más débil que la del núcleo principal, con un borde claro, lo que sugiere una membrana nuclear, que tiene una forma redonda, ubicación intracitoplasmática, diámetro inferior a 1/5 del núcleo principal. (Carrard et al., 2007; Dietz et al., 2000).

La WHO identifica el humo de los cigarrillos como el mayor agente de contaminación ambiental doméstica, lo que demuestra la importancia de su evaluación, ya que las personas pasan el $80 \%$ de su tiempo en interiores, como hogares y trabajo. Este humo incluye más de 4000 sustancias generadas a partir de aproximadamente 600 ingredientes de cigarrillos, muchos de los cuales son tóxicos (Lofroth, 1989). En el humo del cigarrillo ya se han identificado sustancias tóxicas, que tienen acción farmacológica activa, citotóxica, antigénica, mutagénica o cancerígena. La fase gaseosa del humo del cigarrillo (92\%) está compuesta, entre otras cosas, de monóxido de carbono, dióxido de carbono, óxidos de nitrógeno, amoníaco, nitrosaminas volátiles, cianuro, compuestos volátiles que contienen azufre, hidrocarburos volátiles, alcoholes, amonio, cetonas, formaldehído, acetaldehído y acroleína. La fase de partículas representa el 8\% del humo del cigarrillo y contiene agua, nicotina y alquitrán que solo concentra 43 sustancias altamente tóxicas como arsénico, níquel, benzopireno, cadmio y plomo (Lofroth, 1989).

La exposición crónica a este humo causa cambios metaplásicos en la mucosa respiratoria, como un aumento en el número y tamaño de las células caliciformes, en consecuencia, un aumento en la secreción de las vías respiratorias (Mullen et al. 1987). Kreindler et al. (2005) también demostraron in vitro que la exposición al humo del cigarrillo puede inhibir el transporte de cloruro en las células epiteliales, promoviendo cambios fisiológicos similares a los encontrados en pacientes con fibrosis quística.

La educación continua y el desarrollo social frente a la necesidad de la transmisión del conocimiento a la población, reduciría la exposición de estos animales y evitaría problemas futuros a medida que el problema se soluciona. La gestión de la información debe ir más allá de las universidades, teniendo la necesidad de llegar a la población a través de un lenguaje simple y sin complicaciones (Almeida Junior et al., 2019; Junior et al., 2019).

\section{Consideraciones finales}

A partir de lo que se encuentra en la literatura, la prueba de micronúcleos es efectiva para la evaluación no invasiva de la mutagenicidad por las que las células escaladoras de la mucosa oral, presentan un bajo costo, se caracteriza por ser fácil de realizar, leer y confiable. A partir de esta revisión de la literatura, fue posible observar un efecto pro-mutagénico del humo del cigarrillo en la mucosa oral de los perros expuestos al tabaquismo pasivo.

\section{Agradecimientos}

Este estudio fue financiado en parte por la Coordinación de Perfeccionamiento de Personal de Nível Superior - Brasil (CAPES) - Código de Finanzas 001.

\section{Referencias}

Almeida Junior, S., Silva, F. C., Moreira, N. I. T., Bulgo, D. C., Oliveira, L. N., Rodrigues, A. A., ... Andrade, G. 2019. Bases pedagógicas em curso profissionalizante de Farmácia e Laboratório Clínico como apoio na construção profissional do indivíduo. Revista Eletrônica Acervo Saúde, 25, e649.

Almeida Junior, S., Silva, M. S., Popolim, R. S., Goncalves, C. R., Melo, M. R. S., \& Bulgo, D. C. 2019. Dissemination of knowledge and scientific production in professionalizing courses: a report of experience. PubSaúde, 2, a008.

Armour, J., Anderson, D., Amstutz, H., \& Wolf, A. 1998. The Merck Veterinary Manual (8a ed.). Oxford, UK: Merial.

Bascom, R., Kesavanathan, J., Fitzgerald, T. K., Cheng K. H., \& Swift, D. L. 1995. Sidestream tobacco smoke exposure acutely alters human nasal mucociliary clearance. Environ Health Perspect.;103(11), 1026-1030. 
Carrard, V. C., Costa, C. H., Ferreira, L. A., Lauxen, I. S., \& Rados, P. V. 2007. Teste dos micronúcleos: um biomarcador de dano genotóxico em células descamadas da mucosa bucal. Revista da Faculdade de Odontologia de Porto Alegre, 48(1-3), 77-81.

Carvalho, M. B. D., Ramirez, A., Gattás, G. J. F., Guedes, A. L., Amar, A., Rapaport, A., ... Curioni, O. A. 2002. Correlação entre a evolução clínica e a freqüência de micronúcleos em células de pacientes portadores de carcinomas orais e da orofaringe. Revista da Associação Médica Brasileira, 48(4), 317-322.

Dietz, J., Diehl, A. S., Prolla, J. C., \& Furtado, A. D. 2000. Pesquisa de micronúcleos na mucosa esofágica e sua relação com fatores de risco ao câncer de esôfago. Revista da Associação Médica Brasileira, 46(3), 207-211.

Gilson, S. D., \& Page, R. L. 1998. Princípios de Oncologia. Manual Saunders: Clinica de Pequenos Animais. São Paulo, SP: Roca.

Gomes, F. B. C. 2003. Consequências do tabagismo para a saúde. Camara dos deputados. Brasilia, DF, Brasil. Disponível

em: http://bd.camara.leg.br/bd/bitstream/handle/bdcamara/1569/consequencias_tabagismo_gomes.pdf?seq uence $=1.09 / 10 / 2016$

Goorman, N. T., \& Dobson J. M. 1995. The skin and associated tissues. In: R.A.S. White (Ed), Manual of Small Animal Oncology. Shurdington, UK.

INCA, 2015. Instituto Nacional de Câncer José Alencar Gomes da Silva. Coordenação de Prevenção e Vigilância Estimativa 2016: incidência de câncer no Brasil / Instituto Nacional de Câncer José Alencar Gomes da Silva - Rio de Janeiro; Disponível em: www.inca.gov.br/estimativa/2016/estimativa-2016v11.pdf Acesso em 11 de out de 2016

Karaman, M., \& Tek, A. 2009. Deleterious effect of smoking and nasal septal deviation on mucociliary clearance and improvement after septoplasty. American Journal of Rhinology \& Allergy, 23(1), 2-7.

Kreindler, J. L., Jackson, A. D., Kemp, P. A, Bridges, R. J., \& Danahay, H. 2005. Inhibition of chloride secretion in human bronchial epithelial cells by cigarette smoke extract. American Journal of Physiology - Lung Cellular and Molecular Physiology, 288, 894-902.

Lofroth G. 1989. Environmental tobacco smoke: overview of chemical composition and genotoxic components. Mutation Research, 222, 73-80.

Maria, P. P., Sobral, R. A., \& Daleck, C. R. 1998. Casuística de cães portadores de neoplasias atendidos no Hospital Veterinário da Unesp / Jaboticabal durante o período de 01/01/95 a 01/05/97. In: Congresso Brasileiro De Cirúrgia E Anestesiologia Veterinária, 3. 1998, Belo Horizonte. Anais Santa Maria: Colégio Brasileiro de Cirurgia e Anestesiologia Veterinária, 1998. p. 61.

Meireles, J. R. C., Lopes, M. A., Alves, N. N., \& Cerqueira, E. M. M. 2006. Apoptosis in exfoliated cells from the oral mucosa of individuals occupationally exposed to mutagenic and carcinogenic agents. Revista Brasileira de Cancerologia, 52(4), 337-343.

Moralles, E. N. 2014. Estudo dos efeitos mutagênicos do tabagismo passivo em cães. São Paulo, Faculdade de Medicina, Universidade de São Paulo. Dissertação de mestrado.

Morrison, W. B. 1998. Cancer in Dogs and Cats: Medical and Surgical Management. Baltimore, MA: Iowa State University Veterinarian.

Mullen, J. B. M., Wright, J. L., Wiggs, B. R., \&Paré, P. D., \&Hogg, J. C. 1987. Structure of central airways in current smokers and ex-smokers with and without mucus hypersecretion: relationship to lung function. Thorax, 42(11), 843-848.

Rodrigues, A. A., \& Junior, S. A. (2019). Carcinomas de células escamosas e modalidades de tratamento em cães. Atas de Saúde Ambiental, 7, 84-91.

Santos, M. O. 2018. Estimativa 2018: Incidência de Câncer no Brasil. Revista Brasileira de Cancerologia, 64, 119-120.

Schmid, W. 1975. The micronucleus test. Mutation Research, 31.

Silva, I. C., Peron, M. C. C., Arbex, M. A., Lichtenfels, A., Lobo, D., Giocondo, M., ... Soares, C. 2012. Micronucleus formation induced by biomass burning particles derived from biomss burning induce high micronucleus frequency in Tradescantia pallida asssay. Journal of the Brazilian Society of Ecotoxicology, 7(1), 11-17.

Sisenando, H. A., Medeiros, S. R., Saldiva, P. H., Artaxo, P., \& Hacon, S. S. 2011. Genotoxic potential generated by biomass burning in the Brazilian Legal Amazon by Tradescantia Micronucleus bioassay: a toxicity assessment study. Environ Health. 10(41),1-9. 
Stanley, P. J., Wilson, R., Greenstone, M. A., MacWillian, L., \& Cole, P. J. 1986. Effect of cigarette smoking on nasal mucociliary clearance and ciliary beat frequency. Thorax, 41(7), 519-523.

Sturion, D. J., Santos, P. C. G., Santos, R. V., Laganato, S., Abreu, C., Isquerdo, R., \& Garbelini, M. 1997. Osteocondroma em cão Fila Brasileiro. In: Congresso brasileiro de clínicos veterinários de pequenos animais, 1997, Curitiba. Anais. Curitiba: Associação Nacional de Clínicos Veterinários de Pequenos Animais - Paraná. p. 48.

Withrow, S. J., \& Macewen, E. G. 1996. Small Animal Clinical Oncology (2a ed.). Philadelphia, PA: Saunders. Word Health Organization, WHO. 2011. Report in the global tobacco epidemic: warning about the dangers of tobacco. Geneva: Word Health Organization; 2011. pp.152 pp. Ref.162

Wünsch Filho, V., Mirra, A. P., López, R. V. M., \& Antunes, L. F. 2010. Tabagismo e câncer no Brasil: evidências e perspectivas. Revista Brasileira de Epidemiologia, 13(2), 175-187.

\section{Minicurrículo}

Alex Alves Rodrigues. Graduado en Medicina Veterinaria de United Metropolitan College, trabajo como técnico de laboratorio I con enfoque en bioterismo y énfasis en la creación y mantenimiento de conejillos de indias a partir de modelos animales convencionales (ratas y ratones), modelos GMO, control de natalidad de madres, rutina de laboratorio y control. microbiologico Capacitación en áreas relacionadas en el Instituto Butantan.

Jéssica Cardoso Faria. Estudiante del curso de Farmacia y Laboratorio en Euro Anglo Vocational Courses y estudiante de G.E.T.o.S. (Grupo de Estudio e Investigación de Toxicología y Promoción de la Salud).

Andrea Barbosa Pimenta. Graduado en Medicina Veterinaria por la Faculdade Sudoeste Paulista, Avaré, São Paulo, Brasil.

Jorge Alberto Prieto Pérez. Estudiante de último semestre del programa de Comunicación Social Periodismo de la Universidad Pontificia Bolivariana seccional Bucaramanga. Santander. Colombia.

Silvio de Almeida Junior. Doctorando en Promoción de la Salud por la Universidad de Franca (beca CAPES), Master (beca CAPES) en Ciencia Animal por la misma institución siguiendo la línea de investigación de analgesia e inflamación de productos naturales con pruebas biológicas que involucran patología / farmacología. Especialista en Lúdico y Psicopedagogía Clínica e Institucional en la Faculdade Dom Bosco trabajando con el desarrollo de habilidades cognitivas. Graduado en Biomedicina de la Universidad de Franca con iniciación científica en toxicología y mutagénesis. Experiencia clínica y de laboratorio (análisis clínico, histopatología, citología), enseñanza en cursos de salud (Farmacología / Patología / Fisiología), pruebas preclínicas (toxicología, genética, farmacología. Inflamación, analgesia, iNos y estadística). Actualmente, estudiante de posgrado en biomedicina estética en la Faculdade Faveni.

Como citar: Rodrigues, A.A., Faria, J.C., Pimenta, A.B., Perez, J.A.P., Almeida Junior, S. Prueba de micronucleo en mucosa para la evaluación de toxicidad en perros por exposición al tabaco. Pubsaúde, 3, a016. DOI: https:// dx.doi.org/10.31533/pubsaude3.a016

Recebido: 02 nov. 2019.

Revisado e aceito: 08 mar. 2020.

Conflito de interesse: os autores declaram, em relação aos produtos e companhias descritos nesse artigo, não ter interesses associativos, comerciais, de propriedade ou financeiros que representem conflito de interesse.

Licenciamento: Este artigo é publicado na modalidade Acesso Aberto sob a licença Creative Commons Atribuição 4.0 (CC-BY 4.0). 